\title{
Carvedilol Reduces the Severity of Central Sleep Apnea in Chronic Heart Failure
}

\author{
Akira Tamura, MD; Yoshiyuki Kawano, MD; Junichi Kadota, MD
}

\begin{abstract}
Background Carvedilol may reduce the severity of central sleep apnea (CSA) in patients with chronic heart failure (CHF).

Methods and Results This study prospectively examined the effect of carvedilol on the severity of CSA in patients with CHF. Polysomnographic findings, left ventricular (LV) function, and plasma brain natriuretic peptide (BNP) level were evaluated before and 6 months after induction of carvedilol in 16 patients with CHF (New York Heart Association functional class II or III and LV ejection fraction $<50 \%$ ) who had CSA (central apnea index $[\mathrm{CAI}]>5$ with dominant central apneic events). All patients tolerated carvedilol. The 6-month treatment with carvedilol increased the LV ejection fraction $(32 \pm 7.4 \%$ to $45 \pm 9.8 \%, \mathrm{P}<0.001)$ and decreased the BNP level (159 [69-458] pg/ml to 38 [16-193] pg/ml, $\mathrm{P}=0.017)$. The polysomnographic findings showed that the treatment decreased the apnea-hypopnea index ( $34 \pm 13$ to $14 \pm 13, \mathrm{P}=0.003)$ and $\mathrm{CAI}(13 \pm 11$ to $1.9 \pm 4.3, \mathrm{P}<0.001)$, whereas it increased the obstructive apnea index $(1.1 \pm 1.5$ to $3.1 \pm 3.4, \mathrm{P}=0.04)$.

Conclusion This preliminary study shows that treatment with carvedilol reduces the severity of CSA in patients with CHF, but that episodes of obstructive sleep apnea sometimes increase after the treatment. (Circ J 2009;
\end{abstract} 73: 295-298)

Key Words: Carvedilol; Central sleep apnea; Chronic heart failure

$\mathbf{P}$ revious studies have shown that central sleep apnea (CSA) occurs in $30-50 \%$ of patients with chronic heart failure (CHF) due to left ventricular (LV) systolic dysfunction, ${ }^{1-4}$ and that CSA is associated with increased morbidity and mortality in those patients ${ }^{5-8}$ Our study involving 44 patients with CHF recently demonstrated that (1) patients taking $\beta$-blockers (carvedilol) had a lower central apnea index (CAI) than those not taking them, (2) the dose of $\beta$-blockers was negatively correlated with the CAI, and (3) no use of $\beta$-blockers was independently associated with a higher CAI? These results suggest that $\beta$-blockers may suppress CSA in a dose-dependent manner in patients with CHF. Accordingly, we prospectively examined the effect of carvedilol on the severity of CSA in 16 patients with CHF.

\section{Methods}

\section{Study Population}

Eligible patients with stable CHF who met the following criteria were considered for enrollment and underwent overnight polysomnography: age $>20$ years, not being treated with $\beta$-blockers, ischemic or idiopathic dilated cardiomyopathy, stable symptoms of CHF (New York Heart Association functional class II or III) within the preceding 2 months, and an LV ejection fraction (LVEF) $<50 \%$. The exclusion criteria were recent ( $<6$ months) acute coronary syndrome,

(Received July 14, 2008; revised manuscript received September 23, 2008; accepted October 13, 2008; released online December 27, 2008) Internal Medicine 2, Faculty of Medicine, Oita University, Yufu, Japan Mailing address: Akira Tamura, MD, Internal Medicine 2, Faculty of Medicine, Oita University, 1-1 Idaigaoka, Hasama-machi, Yufu 8795593, Japan. E-mail: akira@med.oita-u.ac.jp

All rights are reserved to the Japanese Circulation Society. For permissions, please e-mail: cj@j-circ.or.jp resting angina, other concomitant heart disease, systolic blood pressure $<100 \mathrm{mmHg}$, bradycardia ( $<50$ beats $/ \mathrm{min}$ ), $2^{\text {nd }}$ or $3^{\text {rd }}$ degree atrioventricular block, life-threatening arrhythmia, previous cerebrovascular disease, neurological disease, chronic respiratory disease, Raynaud's phenomenon, and peripheral artery disease. Patients who had a CAI $>5$ with dominant central apneic events on overnight polysomnography were finally enrolled in the study. The study protocol was approved by the institutional ethics committee, and informed consent was given by each patient before the study.

\section{Study Protocol}

All patients began taking carvedilol during hospitalization, initially $1.25 \mathrm{mg}$ twice daily, with the does increasing stepwise at 1 - or 2 -week intervals to reach $10 \mathrm{mg}$ twice daily or the maximum tolerated dose of $<10 \mathrm{mg}$ twice daily. Patients then took carvedilol at a fixed dose. Overnight polysomnography was performed 6 months after induction of carvedilol. In addition, echocardiography and measurement of the plasma BNP level were performed before and 6 months after induction of carvedilol. Other cardiovascular medications were unchanged during the study.

\section{Polysomnography}

Overnight polysomnography was performed using a computerized system (E-series; Compumedics Ltd, Abbotsford, Vic., Australia) that consisted of monitoring of the electroencephalogram, electro-oculogram, submental electromyogram, electrocardiogram, chest and abdominal movement using respiratory effort bands, oronasal airflow by an airflow pressure transducer, and arterial oxyhemoglobin saturation $\left(\mathrm{SpO}_{2}\right)$ by pulse oximetry. A central apnea was defined as the absence of oronasal airflow for $\geq 10$ s associated with an absent inspiratory effort. An obstructive apnea was defined as the absence of oronasal airflow for $\geq 10$ s associated with 
Table 1 Patients' Characteristics

\begin{tabular}{lc}
\hline \hline$n$ & 16 \\
Age (years) & $63 \pm 15$ \\
Men & $12(75)$ \\
Body mass index $\left(\mathrm{kg} / \mathrm{m}^{2}\right)$ & $23 \pm 3.0$ \\
Hypertension & $12(75)$ \\
Diabetes mellitus & $3(19)$ \\
Current smoker & $1(6)$ \\
Dyslipidemia & $14(88)$ \\
Basic heart disease & \\
Idiopathic dilated cardiomyopathy & $13(81)$ \\
Previous myocardial infarction & $3(19)$ \\
Chronic atrial fibrillation & $1(6)$ \\
NYHA functional class & \\
II & $1(6)$ \\
III & $15(94)$ \\
LVEF (\%) & $32 \pm 7.4$ \\
BNP (pg/ml) & $159(69-458)$ \\
Polysomnography & \\
AHI & $34 \pm 13$ \\
CAI & $13 \pm 11$ \\
OAI & $1.1 \pm 1.5$ \\
Loop diuretics & $15(94)$ \\
Spironolactone & $13(81)$ \\
ACEI & $2(13)$ \\
ARB & $10(63)$ \\
Digitalis & $3(19)$ \\
&
\end{tabular}

Data are mean $\pm S D$, median (first-third quartiles), or $n(\%)$.

NYHA, New York Heart Association; LVEF, left ventricular ejection fraction; $B N P$, brain natriuretic peptide; AHI, apnea-hypopnea index; CAI, central apnea index; OAI, obstructive apnea index; ACEI, angiotensin-converting enzyme inhibitor; $A R B$, angiotensin-receptor blocker. continued or increased inspiratory effort. A hypopnea was defined as $\geq 50 \%$ reduction in oronasal airflow for $\geq 10 \mathrm{~s}$ associated with $\geq 3 \%$ fall in $\mathrm{SpO}_{2}$. The apnea-hypopnea index (AHI) was calculated as the mean number of apneic and hypopneic episodes per hour of sleep. In addition, the CAI or obstructive apnea index (OAI) was calculated as the mean number of central or obstructive apneic episodes, respectively. Since a central hypopnea cannot be easily distinguished from an obstructive hypopnea, ${ }^{10}$ CAI was used as an indicator of CSA.

\section{Echocardiography}

Echocardiography was performed using the standard techniques. The LVEF was calculated using a modification of Simpson's rule!1

\section{Measurement of Plasma Brain Natriuretic Peptide (BNP) Level}

The plasma BNP level was measured using a specific immunoradiometric assay (Shinoria BNP kit; Shionogi Co Ltd, Osaka, Japan).

\section{Statistical Analysis}

The data are expressed as the means \pm SD, median (firstthird quartiles), or number (\%). Continuous variables were analyzed by the paired t-test or the Wilcoxon signed-rank sum test. Spearman's rank correlations were used to examine any correlations between the continuous variables. A P-value $<0.05$ was considered statistically significant. All analyses
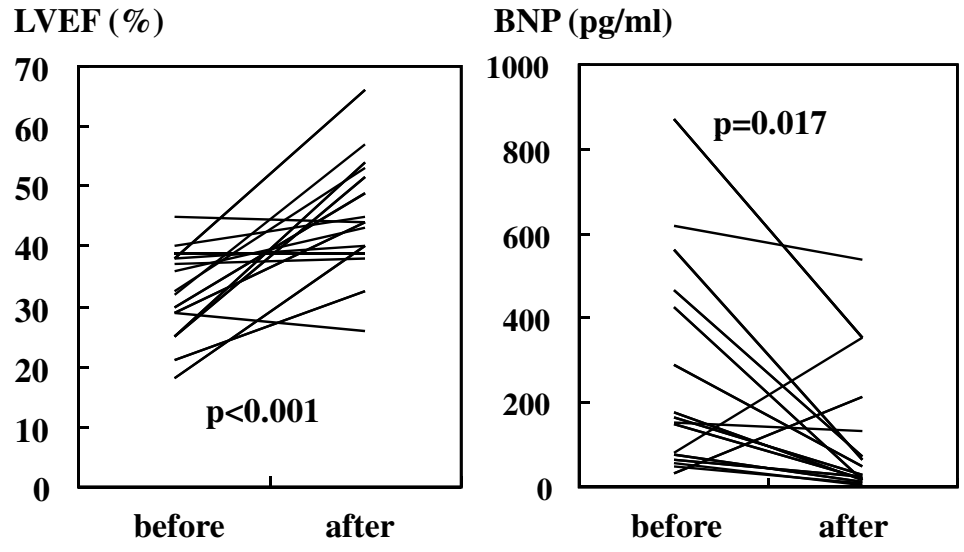

Fig 1. Changes in the left ventricular ejection fraction (LVEF) and the brain natriuretic peptide (BNP) levels before and 6 months after induction of carvedilol.
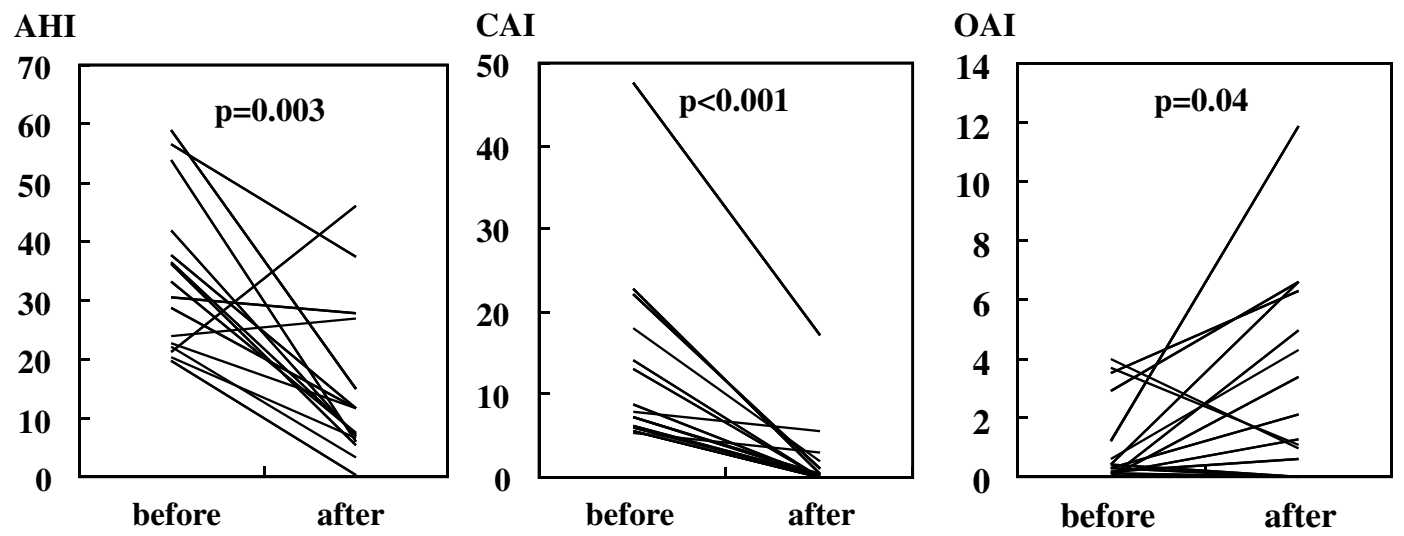

Fig 2. Changes in the apnea-hypopnea index (AHI), central apnea index (CAI), and obstructive apnea index (OAI) before and 6 months after induction of carvedilol. 

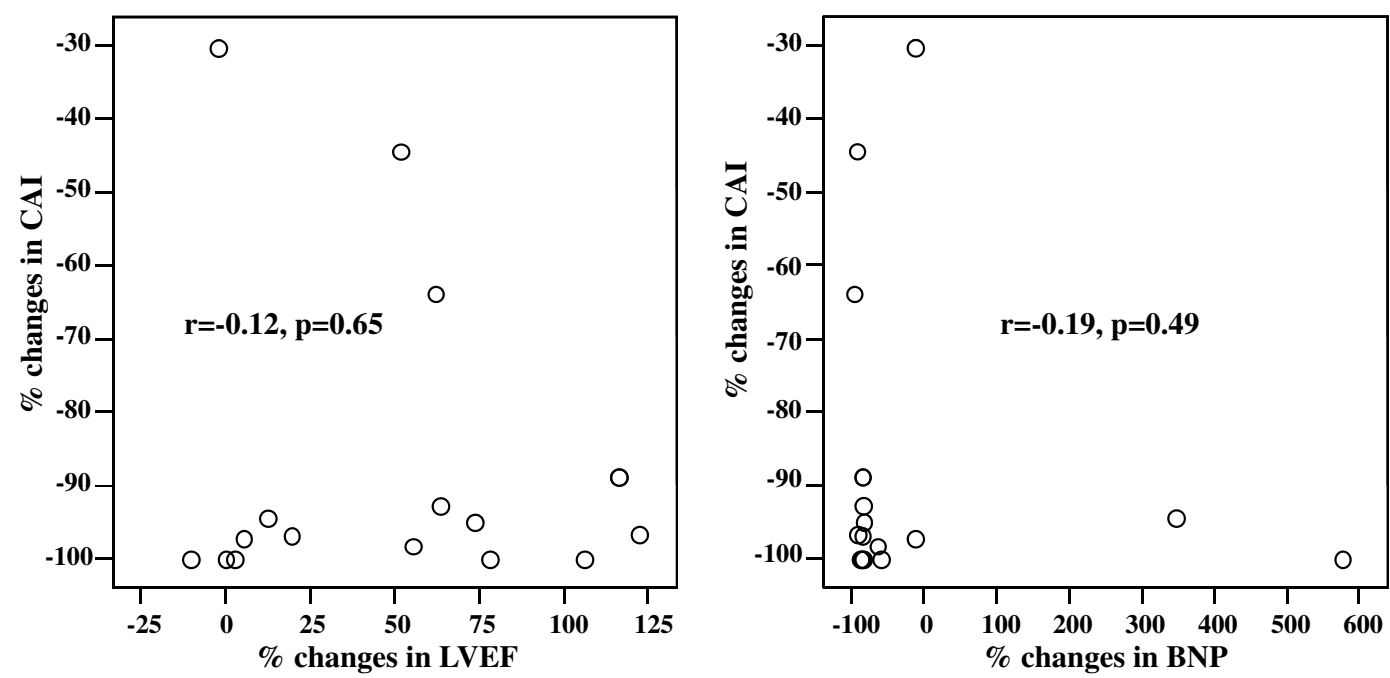

Fig 3. Associations of $\%$ changes in the central apnea index $(\mathrm{CAI})$ or $\%$ changes in left ventricular ejection fraction (LVEF) before and 6 months after induction of carvedilol and \% changes in plasma brain natriuretic peptide (BNP) levels before and 6 months after the induction.

were performed using SPSS 12.0J for Windows (SPSS Inc, Tokyo, Japan).

\section{Results}

A total of 16 patients (12 men, 4 women, age $63 \pm 15$ years) were enrolled and their characteristics are shown in Table 1. The etiology of CHF was idiopathic dilated cardiomyopathy in 13 patients and previous myocardial infarction in 3 patients. The frequency of hypertension, diabetes, current smoker, and dyslipidemia was $75 \%, 19 \%, 6 \%$, and $88 \%$, respectively. Loop diuretics, spironolactone, angiotensin-converting enzyme inhibitors, angiotensin-receptor blockers, and digitalis had been prescribed for $94 \%, 81 \%$, $13 \%, 63 \%$, and $19 \%$ of the patients, respectively.

All patients tolerated carvedilol; the maintenance dose ranged from 10 to $20 \mathrm{mg} /$ day. There were no significant differences in the body mass index or total sleep time before and 6 months after induction of carvedilol $\left(23 \pm 3.0 \mathrm{~kg} / \mathrm{m}^{2} \mathrm{vs}\right.$ $23 \pm 3.4 \mathrm{~kg} / \mathrm{m}^{2}$ and $332 \pm 83 \mathrm{~min}$ vs $340 \pm 65 \mathrm{~min}$, respectively). Treatment with carvedilol increased the LVEF significantly $(32 \pm 7.4 \%$ to $45 \pm 9.8 \%, \mathrm{P}<0.001)$ and decreased the BNP level significantly $(159[69-458] \mathrm{pg} / \mathrm{ml}$ to $38[16-193] \mathrm{pg} / \mathrm{ml}$, $\mathrm{P}=0.017)$ (Fig 1). The treatment decreased the AHI and CAI significantly $(34 \pm 13$ to $14 \pm 13, P=0.003$ and $13 \pm 11$ to $1.9 \pm 4.3, \mathrm{P}<0.001$, respectively), and increased the OAI significantly $(1.1 \pm 1.5$ to $3.1 \pm 3.4, \mathrm{P}=0.04)$ (Fig 2). The $\%$ changes in the CAI before and 6 months after induction of carvedilol did not correlate significantly with either the \% changes in the LVEF or the \% changes in the BNP levels before and 6 months after induction $(\mathrm{r}=-0.12, \mathrm{P}=0.65$ and $\mathrm{r}=-0.19, \mathrm{P}=0.49$, respectively) $($ Fig 3).

\section{Discussion}

Little information is available regarding the effects of standard medical therapy on CSA in patients with CHF. Tremel et al reported that the AHI did not change significantly after 2 months of optimal medical treatment in 15 patients who had been diagnosed as having CSA on polysomnography at 1 month after recovering from acute pul- monary edema caused by LV systolic failure! ${ }^{2}$ Their results contradict the results of the present study, which indicate that carvedilol reduces the severity of CSA in patients with $\mathrm{CHF}$. The exact reasons for this discrepancy are unclear, but 2 months of medical treatment might be too short to evaluate the long-term effect of the treatment on CSA in patients with CHF. In addition, there was no description of the use or dose of $\beta$-blockers in that study.

The exact mechanisms by which carvedilol reduces the severity of CSA in patients with CHF are unclear, but the following are assumed. First, carvedilol could restore the enhanced central chemosensitivity to $\mathrm{CO}_{2}$, resulting in suppression of CSA in patients with CHF. It is thought that enhanced central chemosensitivity to $\mathrm{CO}_{2}$ plays a major role in the genesis of CSA in patients with CHF 13 In a study involving 6 healthy volunteers, Takahashi et al demonstrated that intravenous administration of isoproterenol increases the hypercapneic ventilatory response, which is an index of the central chemosensitivity to $\mathrm{CO}_{2}$, and that intravenous administration of propranolol decreases the response ${ }^{14}$ However, there is no evidence that carvedilol indeed restores the enhanced central chemosensitivity to $\mathrm{CO}_{2}$ in CHF patients with CSA. Therefore, further investigations are required to clarify this point. Second, carvedilol could reduce the severity of CSA through its favorable effects on LV function in patients with CHF. Pulmonary vagal afferent nerve stimulation caused by pulmonary congestion results in brief central apnea followed by tachypnea and hyperventilation, contributing to the genesis of CSA in patients with CHF ${ }^{15-18}$ Prolonged circulation time between the lungs and the chemoreceptors because of low cardiac output affects the length of both the hyperpneic phase and the total periodic breathing cycle ${ }^{19,20}$ contributing to the maintenance of CSA in patients with CHF. Therefore, improvement in LV function could lead to a reduction in the severity of CSA. Solin et al demonstrated a significant reduction in the AHI associated with a significant fall in pulmonary capillary wedge pressure after intensive medical and/or nasal continuous positive airway pressure therapy in 7 patients with CHF and CSA, although they did not investigate the association between changes in the $\mathrm{AHI}$ and those 
in pulmonary capillary wedge pressure before and after therapy $!^{18}$ In the present study, the \% changes in the CAI before and 6 months after induction of carvedilol did not correlate significantly with either the $\%$ changes in the LVEF or the \% changes in BNP level before and after the treatment. However, the sample size of the present study was very small, so it cannot be denied that the improvement in LV function is associated with a reduction in the severity of CSA. The association between the reduction in the severity of CSA and the improvement in the indexes of cardiac function, including pulmonary capillary wedge pressure, remains to be further investigated.

Of interest, in the present study, treatment with carvedilol increased the OAI significantly, although the exact mechanisms for this phenomenon are unclear. Preexisting obstructive sleep apnea that had been masked by CSA might have appeared after the treatment and this phenomenon requires further detailed investigation.

\section{Study Limitations}

First, the sample size was very small, so further studies with a large population are needed to confirm the results. Second, we did not have a control group (ie, patients without carvedilol treatment). Because $\beta$-blocker treatment is already established as a standard therapy for CHF, no treatment with $\beta$-blockers for patients with CHF would not be ethically allowed. Third, the present study included patients with stable symptoms of CHF within the preceding 2 months of the study. The registration of patients with a longer period of stable symptoms would be more optimal: the natural time course of CHF or the therapeutic history for CHF may have, to a lesser degree, affected the results of the present study. Fourth, it is unclear whether other types of $\beta$-blockers are as effective against CSA as carvedilol. Finally, the dose of carvedilol used in the present study was smaller than that generally used in Western countries. However, the MUCHA trial demonstrated that $5-20 \mathrm{mg} /$ day of carvedilol is recommended for the treatment for Japanese patients with CHF ${ }^{21}$ This difference in dosage is assumed to be because $\beta 1$-receptor sensitivity is higher in Asian than in Caucasians or African-Americans?22

\section{Conclusions}

The present study shows that treatment with carvedilol reduces the severity of CSA in patients with CHF, but that episodes of obstructive sleep apnea sometimes increase after the treatment.

\section{References}

1. Naughton MT, Benard DC, Liu PP, Rutherford R, Rankin F, Bradley TD. Treatment of congestive heart failure and Cheyne-Stokes respiration during sleep by continuous positive airway pressure. Am J Resir Crit Care Med 1995; 151: 92-97.

2. Javaheri S, Parker TJ, Wexler L, Michaels SE, Stanberry E, Nishyama $\mathrm{H}$, et al. Occult sleep-disordered breathing in stable congestive heart failure. Ann Intern Med 1995; 122: 487-492.

3. Javaheri S, Parker TJ, Liming JD, Corbett WS, Nishiyama H, Wexler $\mathrm{L}$, et al. Sleep apnea in 81 ambulatory male patients with stable heart failure: Types and their prevalences, consequences, and presentations. Circulation 1998; 97: 2154-2159.

4. Sin DD, Fitzgerald F, Parker JD, Newton G, Floras JS, Bradley TD. Risk factors for central and obstructive sleep apnea in 450 men and women with congestive heart failure. Am J Respir Crit Care Med 1999; 160: 1101-1106.

5. Hanly PJ, Zuberi-Khokhar NS. Increased mortality associated with Cheyne-Stokes respiration in patients with congestive heart failure. Am J Respir Crit Care Med 1996; 153: 272-276.

6. Wilcox I, McNamara SG, Wessendorf T, Willson GN, Piper AJ, Sullivan CE. Prognosis and sleep disordered breathing in heart failure. Thorax 1998; 53(Suppl 3): S33-S36.

7. Lanfranchi PA, Braghiroli A, Bosimini E, Mazzuero G, Colombo R, Donner CF, et al. Prognostic value of nocturnal Cheyne-Stokes respiration in chronic heart failure. Circulation 1999; 99: 1435-1440.

8. Sin DD, Logan AG, Fitzgerald FS, Liu PP, Bradley TD. Effects of continuous positive airway pressure on cardiovascular outcomes in heart failure patients with and without Cheyne-Stokes respiration. Circulation 2000; 102: 61-66.

9. Tamura A, Kawano Y, Naono S, Kotoku M, Kadota J. Relationship between $\beta$-blocker treatment and the severity of central sleep apnea in chronic heart failure. Chest 2007; 131: 130-135.

10. Iber C, Ancolo-Israel S, Chesson AL Lr, Quan SF for the American Academy of Sleep Medicine. The AASM manual for the scoring of sleep and associated events: Rules, teminology and technical specifications. AASM; 2007; 1-59.

11. Folland ED, Parisi AF, Moynihan PF, Jones DR, Feldman CL, Tow DE. Assessment of left ventricular ejection fraction and volumes by real-time, two-dimensional echocardiography. Circulation 1979; 60: $760-766$.

12. Tremel F, Pépin JL, Veale D, Wuyam B, Siché JP, Mallion JM, et al. High prevalence and persistence of sleep apnoea in patients referred for acute left ventricular failure and medically treated over 2 months. Eur Heart J 1999; 20: 1201-1209.

13. Javaheri S. A mechanism of central sleep apnea in patients with heart failure. $N$ Engl J Med 1999; 341: 949-954.

14. Takahashi T, Shinozaki T, Sugimura K, Ogawa H, Okabe S, Watanabe J, et al. Impact of beta-stimulation to central chemoreceptor sensitivity (abstract). Circ J 2004; 68(Suppl I): 138.

15. Churchill ED, Cope O. The rapid shallow breathing resulting from pulmonary congestion and edema. J Exp Med 1929; 49: 531-537.

16. Paintal AS. Mechanism of stimulation of type J pulmonary receptors. J Physiol 1969; 203: 511-532.

17. Roberts AM, Bhattacharya J, Schultz HD, Coleridge HM, Coleridge JC. Stimulation of pulmonary vagal afferent $\mathrm{C}$-fibers by lung edema in dogs. Circ Res 1986; 58: 512-522.

18. Solin P, Bergin P, Richardson M, Kaye DM, Walters EH, Naughton MT. Influence of pulmonary capillary wedge pressure on central apnea in heart failure. Circulation 1999; 99: 1574-1579.

19. Hall MJ, Xie A, Rutherford R, Ando S, Floras JS, Bradley TD. Cycle length of periodic breathing in patients with and without heart failure. Am J Respir Crit Care Med 1996; 154: 376-381.

20. Mortara A, Sleight P, Pinna GD, Maestri R, Capomolla S, Febo O, et al. Association between hemodynamic impairment and Cheyne-Stokes respiration and periodic breathing in chronic stable congestive heart failure secondary to ischemic or idiopathic dilated cardiomyopathy. Am J Cardiol 1999; 84: 900-904.

21. Hori M, Sasayama S, Kitabatake A, Toyo-oka T, Handa S, Yokoyama $\mathrm{M}$, et al. Low-dose carvedilol improves left ventricular function and reduces cardiovascular hospitalization in Japanese patients with chronic heart failure: The Multicenter Carvedilol Heart Failure Dose Assessment (MUCHA) trial. Am Heart J 2004; 147: 324-330.

22. Xie HG, Kim RB, Wood AJJ, Stein CM. Molecular basis of ethnic differences in drug disposition and response. Annu Rev Pharmacol Toxicol 2001; 41: 815-850. 